\title{
Noise exposure-induced intestinal flora dysbiosis disrupts homeostasis of oxi-inflamm-barrier in the gut-brain axis of APP/PS1 mice: implications for early onset Alzheimer's disease
}

Bo Cui ( $\square$ iamcuib@sina.com)

Tianjin Institute of Environmental and Operational Medicine https://orcid.org/0000-0001-6869-2523 Huimin Chi

Tianjin institute of environmental and operational medicine

\section{Wa Cao}

Tianjin institute of environmental and operational medicine

\section{Donghong Su}

Tianjin institute of environmental and operational medicine Honglian Yang

Tianjin institute of environmental and operational medicine Zhe Li

Tianjin institute of environmental and operational medicine

\section{Xiaojun She}

Tianjin institute of environmental and operational medicine

\section{Kun Wang}

Tianjin institute of environmental and operational medicine

\section{Xiujie Gao}

Tianjin institute of environmental and operational medicine

\section{Kefeng Ma}

Tianjin institute of environmental and operational medicine

\section{Research article}

Keywords: environmental noise, early onset Alzheimer's disease (EOAD), oxidative stress, inflammation, microbiome-gut-brain axis, APP/PS1 mouse

Posted Date: April 6th, 2020

DOI: https://doi.org/10.21203/rs.3.rs-19045/v1 
License: (c) (i) This work is licensed under a Creative Commons Attribution 4.0 International License. Read Full License 


\section{Abstract}

Background: Environmental noise exposure and genetic risk factors are thought to be associated with gut microbiome that exacerbates Alzheimer's disease (AD) pathology. However, the role and mechanism of environmental risk factors in early-onset $A D(E O A D)$ pathogenesis remain unclear.

Methods: We established APP/PS1 Tg and C57BL/6 (wild type [WT]) mouse models to evaluate the molecular pathways underlying EOAD pathophysiology following environmental noise exposure. $16 \mathrm{~S}$ rRNA sequencing analyses were used for intestinal flora measurements and Tax4Fun were used to predict the metagenome content from 16S rRNA sequencing results; and assessment of the flora dysbiosis-triggered dyshomeostasis of oxi-inflamm-barrier and the effects of the CNS end of the gutbrain axis were conducted to explore the underlying pathological mechanisms.

Results: Both WT and APP/PS1 mice showed statistically significant relationship between environmental noise and the taxonomic composition of the corresponding gut microbiome. Bacterial-encoded functional categories in noise-exposed WT and APP/PS1 mice included phospholipid and galactose metabolism, oxidative stress, and cell senescence. These alterations corresponded with imbalanced intestinal oxidation and anti-oxidation systems and low-grade systemic inflammation after noise exposure. Mechanistically, axis-series experiments demonstrated that after noise exposure, intestinal and hippocampal tight junction proteins levels reduced, whereas serum levels of inflammatory mediator were elevated. With regard to APP/PS1 overexpression, noise-induced abnormalities in the gut-brain axis may contribute to aggravation of neuropathology in the presymptomatic stage of EOAD mice model.

Conclusions: Our results demonstrate that noise exposure has deleterious effects on the homeostasis of oxi-inflamm-barrier in the microbiome-gut-brain axis. Therefore, at least in a genetic context, chronic noise may aggravate the progression of EOAD.

\section{Background}

Presently, the pathogenesis of Alzheimer's disease (AD) is generally considered to involve the synergistic effects of internal (genetic) and external environmental factors [1-2]. Early-onset Alzheimer's disease $(E O A D)$ is a rare form of neurodegeneration disease with a large genetic basis that is only partially understood. Only a limited proportion of EOAD cases are linked to autosomal dominant mutations in the amyloid precursor protein (APP), presenilin 1 (PSEN1), or presenilin 2 (PSEN2) genes [3]. One possible explanation is that environmental factors may promote $A D$ pathogenesis. For example, early-life stress has a long-lasting effect on the neuroinflammatory response in APP/PS1 mice, a transgenic mouse model of EOAD, and may aggravate the neuropathology and alter disease progression [4].

Noise is one of the most important environmental health hazards, which causes mental stress associated with adverse health effects [5]. Exposure to environmental noise may be associated with behavioral and cognitive deficit both in children [6] and experimental animals [7, 8]. Environmental noise stress has also been associated with $A D$-like cognitive dysfunction as well as persistent tau and amyloid- $\beta$ (A $\beta$ ) 
pathology both in wild type and senescence-accelerated murine model [9-13]. Furthermore, our recent study showed that environmental noise alone or in combination with ApoE4, one of the most important internal risk factors for sporadic late-onset $A D$, can aggravate the occurrence and development of $A D$ [14]. This indicates that the combined effects of environmental noise and genetic risk factors may have a greater impact on EOAD progression than exposure to individual factors alone. Existing studies have indicated that environmental stress may activate the APP/PS1 gene, which may further cause cognitive deficits and impaired neuroinflammatory response to amyloid pathology $[4,15]$. However, the role of environmental noise on the pathological progress of EOAD and its triggering mechanisms remain unclear.

Environmental noise exposure induces a stress reaction through activation of the hypothalamus-pituitaryadrenal (HPA) axis or the sympathetic nervous system (SNS), leading to oxidative stress, inflammation, and endothelial dysfunction[16], which subsequently accelerates the development of cerebrocardiovascular risk factors and disease $[17,18]$. Gut microbiota dysbiosis may have both causal and consequent links to oxidative stress, underlying several neurodegenerative disorders, including $A D$ $[19,20]$. We previously demonstrated that chronic noise-exposure induced stress negatively affects the composition and diversity of the gut microbiota and impairs barrier functions in the intestine and hippocampus [21]. In contrast, oxidative stress in the CNS is currently regarded as a key player in the pathogenesis of AD. Accordingly, we hypothesized that the dysbiosis of gut flora caused by internal and external environmental hazards would induce intestinal oxidative stress, which would subsequently destroy the epithelial barrier function of the gut-brain axis and promote the occurrence and development of AD-like pathological changes.

The present study aimed to evaluate the gut and neuro-molecular pathways involved in EOAD pathophysiology after environmental stress. Based on the results of our previous study, we particularly focused on chronic noise exposure and APP/PS1 overexpression, both of which can trigger the onset of EOAD pathology in a pre-symptomatic stage of the disease at 3 months of age in APP/PS1 mouse model [22]. Moreover, we explored early biochemical and molecular pathological clues that might permit better understanding of the link between EOAD and environmental risks. We used the APP/PS1 double transgenic mouse, a genetically modified model that mimics the human familial AD pathology, to investigate the effects of environmental noise exposure on microbiota-gut-brain axis, oxidative and inflammatory state, and AD-like neuropathology.

\section{Experimental Procedures}

\section{Animals and experimental groups}

Fifty-three 60-day-old male B6C3-Tg (APPswe, PSEN1dE9)/NJU (APP/PS1) and C57BL/6Nju (wild type, WT) mice used in this study were purchased from Nanjing Biomedical Research Institute. To avoid the cage effect from microbiome transfer [23], each mouse was housed in individually ventilated cages under specific pathogen-free conditions, with a 12-hour light/dark cycle (lights on at 06:00) in a temperaturecontrolled room maintained at $23 \pm 2{ }^{\circ} \mathrm{C}$ and a relative humidity of $45-55 \%$. Mice had free access to food 
(Laboratory animals - Nutrients for formula feeds, GB 14924.3-2010) and water in their cages and were habituated to the conditions for 5 days before the start of the experiment.

All APP/PS1 and WT mice were randomly divided into four groups ( $n=10-15$ in a group), with a $2 \times 2$ factor design (independent factors: exposure and genotype). The noise (WT mice) and noise + APP/PS1 (APP/PS1 mice) groups were exposed to white noise at $98 \mathrm{~dB} \mathrm{SPL} \mathrm{(4} \mathrm{h/day} \mathrm{for} 30$ days from 8:00 to 12:00). The control mice (WT mice) and APP/PS1 mice were placed in another room with background noise ( $<40 \mathrm{~dB}$ SPL). After 30 consecutive days of exposure, all animals in this study were sacrificed. Hippocampus tissue, blood, cecal tissue, and their contents were collected immediately for biochemical analyses and stored at $-80^{\circ} \mathrm{C}$ until use. All animal experiments were approved by the Animal and Human Use in Research Committee of the Tianjin Institute of Environmental and Operational Medicine.

\section{Noise exposure set-up}

Noise exposure was conducted as described in our previous research [24]. Briefly, white noise was generated using a sound signal generator (BK 3560C, B\&K Instruments, Denmark), amplified with a power amplifier, and delivered through a loudspeaker. The frequency spectrum of the sound signal from the generator was in the range of 20-20 k Hz. Mice were placed at the center of the sound field and exposed to white noise in a reverberation chamber in wire-mesh cages, where the loudspeaker was suspended directly above the cages.

\section{Sequencing of the $16 \mathrm{~S}$ rRNA gene in microbiota}

We performed 16S rRNA gene sequencing as in our previous research [21]. Briefly, DNA was extracted using the cetyltrimethylammonium bromide/sodium dodecyl sulfate method from cecal contents. Distinct regions of 16S rRNA genes were amplified with specific primers (e.g., 16S V4: 515F-806R, 18S V4: 528F-706R, and 18S V9: 1380F-1510R) with the barcode. PCR reactions were performed with Phusion ${ }^{\circledR}$ High-Fidelity PCR Master Mix (New England Biolabs) and were analyzed to evaluate bacterial diversity using Illumina Hiseq (Novogene Bioinformatics Technology, Beijing, China). Sequences were analyzed using Quantitative Insights Into Microbial Ecology software (http://qiime.org/). A representative sequence was selected for each operational taxonomic unit (OTU) and the taxonomic information for each OTU was annotated by using Ribosomal Database Project classifier.

\section{Taxonomic and functional analysis}

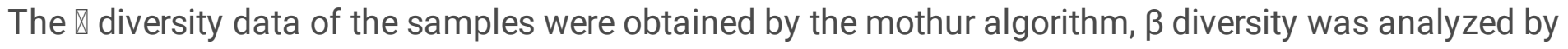
UniFrac distance, and LEFSE (linear discriminant analysis coupled with effect size measurements) was used to identify microbial gene characteristics or microbial-related functional pathways among groups. Linear discriminant analysis (LDA) was used to evaluate the statistical efficiency of biomarkers. In order to further understand the specific functions of each group of bacteria, the $16 \mathrm{~S}$ high-throughput sequencing data were used to classify the OTUs through the SILVAngs platform based on the SILVA database. The $16 \mathrm{~S}$ copy number was then standardized according to the National Center of Biotechnology Information genome annotation. Finally, the prediction of the microbial community 
function was realized by constructing a linear relationship between the SILVA classification and pronuclear classification in the KEGG database.

Analyses for T-AOC, GST, CAT, GSH-Px, and SDH activity

Total antioxidant capacity (T-AOC, No. A015-2-1, ABTS method) and activities of catalase (CAT, No. A0071-1, Visible light method), glutathione S-transferase (GST, No.A004-1-1, Colorimetric method), and glutathione peroxidase (GSH-Px, No. A005-1-2, Colorimetric method) in the cecal contents and succinate dehydrogenase (SDH, No.A022-1-1, colorimetric method) in the colon tissue were analyzed using commercial assay kits (Jiancheng Institute of Biotechnology, Nanjing, China). Detailed operations were performed according to the manufacturer's product manual. The mean value of duplicate samples was taken as the final result for each mouse.

\section{Determination of gene expression by real-time PCR}

Real-time PCR was performed as previously described [14]. Colon tissue from the exposure and control group were homogenized using a rapidly oscillating masher. Total RNA was extracted using an RNeasy kit and then converted to CDNA via reverse transcription using a transcriptor first strand cDNA synthesis kit according to the manufacturer's product manual (TaKaRa Bio, Dalian, China). Specific primers and probes designed for mouse GCLC, GSS, GSR, GSTpi, NOX2/4 (NADPH oxidase 2/4) and GAPDH were used as described in Table 1, and GAPDH gene was used as an internal reference standard. Gene expression was assessed by quantitative real-time PCR under the following thermal cycling conditions: 2 min at $50^{\circ} \mathrm{C}$ and $10 \mathrm{~min}$ at $95^{\circ} \mathrm{C}$ followed by 45 cycles of $95^{\circ} \mathrm{C}$ for $5 \mathrm{~s}$ and $57^{\circ} \mathrm{C}$ for $30 \mathrm{~s}$. Real-time PCR was performed by using gene expression assays-on-demand and a Takara PCR thermal cycler dice real-time system (TaKaRa Bio, Dalian, China). mRNA levels were calculated after normalizing the Ct value to GAPDH expression and are presented as fold-induction values $\left(2^{-\Delta \Delta C t}\right)$ relative to those of WT control mice.

Tab. 1 Primers sequence used for Real-time PCR

\begin{tabular}{cc}
\hline Gene & \multicolumn{1}{c}{ Primers } \\
\hline GCLC & F:5'-CTACCACGCAGTCAAGGACC-3' \\
& R:5'-CCTCCATCAGTAACAACTGGAC-3' \\
GSS & F:5'-AAAGCAGGCCATAGACAGGG-3' \\
& R:5'-TGAATGGGGCATACGTCACC-3' \\
GSR & F:5'-GCGTGAATGTTGGATGTGTACC-3' \\
& R:5'-GTTGCATAGCCGTGGATAATTC-3' \\
GSTpi & F:5'-ATGCCACCATACACCATTGTC-3' \\
& R:5'-GGGAGCTGCCCATACAGAC-3' \\
\multirow{2}{*}{ Nox2 } & F:5'-TGTTTCATTCCTCATCAGAAG-3' \\
\multirow{2}{*}{ Nox4 } & R:5'-CCAACCACACCAGAATGACA-3' \\
& R:5'-TCTGGAAAACCTCCTGCTG-3' \\
GAPDH & F:5'-AGGTCGGTGTGAACGGATTTG-3' \\
& R:5'-TGTAGACCATGTAGTTGAGGTCA-3' \\
\hline
\end{tabular}

\section{Enzyme-linked immunosorbent assay (ELISA)}


Colon and hippocampus tissues for ELISA measurements were homogenized in phosphate buffer ( $\mathrm{PH}=$ 7.4), the lysates were centrifuged at $3000 \mathrm{rpm}$ for $30 \mathrm{~min}$ at $2-8^{\circ} \mathrm{C}$, and the supernatant was collected. The BCA protein quantification assay kit (Thermofisher Scientific) was used to determine the protein concentrations. Levels of ApoA4, interleukin (IL)-6, inducible nitric oxide synthase (INOS), nuclear factor KB (NF-KB), neutrophils gelatinase-associated lipid delivery protein (NGAL), and NF-E2-related factor 2 (Nrf2) in the intestine; levels of $A \beta 40$ and $A \beta 42$ in the hippocampus; and blood plasma levels of $5-\mathrm{HT}$, GABA, D-lactose (D-LAC), endotoxin, IL-6, and tumor necrosis factor (TNF) were measured with mouse ELISA kits (BlueGene Biotech, Shanghai, China) strictly according to the manufacturer's protocol, and chromogenic substrate (tetramethylbenzidine, TMB) and stop solution were used as blank. The average of duplicate samples was taken as the final result for each mouse.

\section{Western blot analysis}

For immunoblot analysis, frozen colon and hippocampus tissues were homogenized and centrifuged as previously described [21] using mouse monoclonal antibodies against phospho-Tau at sites Ser202, Thr205 (AT8, 1:1000, Invitrogen, USA) and rabbit antibodies against the following proteins: phospho-Tau (Ser404) (1:200, Santa Cruz, USA), claudin 1 (CLDN1,1:1,000, Bioworld, USA), occludin (1:800, Bioworld, USA), tight junction protein 1(ZO-1,1:500, Proteintech, USA), and glyceraldehyde phosphate dehydrogenase (GAPDH, 1:10,000, Proteintech, USA). GADPH was used as an internal reference standard. Peroxidase-conjugated goat anti-rabbit and goat anti-mouse IgG $(\mathrm{H}+\mathrm{L})$ (ZSGB-BIO, China) were used as a secondary antibody.

\section{Statistical analysis}

Data presented in graphs indicate the group mean \pm standard deviation and were analyzed by using SPSS version 19.0 and Graphpad Prism 6. Data of REDOX enzyme activity, ELISA, Western blot, and gene expression were analyzed with two-way ANOVA (noise exposure and genotype). The post-hoc analyses were performed using Bonferroni tests for multiple comparison when a significant interaction effect was detected. Data of the 16S rRNA gene analysis were assessed by using Wilcoxon rank sum tests. Redundancy analysis was performed using CANOCO 4.5. Data were considered statistically significant when $\mathrm{P}$ was $<0.05$.

\section{Results}

\section{Chronic noise exposure alters community composition of gut microbiota in APP/PS1 mice}

We assessed mouse gut microbiota structure by $16 \mathrm{~S}$ sequencing-based analysis of gDNA extracted from cecal contents. There were no significant differences in $₫$-diversity or $\beta$-diversity $(P>0.05)$ of the microbial community between noise-exposed and control groups in both WT and APP/PS1 mice; however, the microbiota community in noise-exposed or APP/PS1 mice exhibited less intragroup diversity than that in WT control mice (Fig. 1A, B). At the family level, Muribaculaceae, Lachnospiraceae, Bacteroidaceae, 
Rikenellaceae, Helicobacteraceae, Ruminococcaceae, Bifidobacteriaceae, Lactobacillaceae, Prevotellaceae, and Desulfovibrionaceae were dominant bacteria taxa (Fig. 1C, D). Notably, taxonomic profiling showed that the relative abundance of Muribaculaceae was decreased, while Bacteroidaceae increased markedly (Fig. 1D), which demonstrated that the gut microbiota pattern was markedly altered in chronic noise exposure and APP/PS1 mice.

LEfSe and Metastat analysis was further used to assess species with significant differences between groups based on species abundance after chronic noise exposure. The structure and predominant bacteria of microbiota in each group were represented as a cladogram, and the greatest difference in taxa from phylum to genus level was identified via LDA score (Fig. 2A,

B). At the genus level, species with significant differences between groups were represented as a heatmap (Fig. 2C). Noise exposure significantly elevated levels of Staphylococcus and Papillibacter in the WT groups, and decreased levels of Rikenella and Anaerovorax in mouse model of EOAD (Fig. 2D-J).

\section{Prediction of microbiota functional capacity via Tax4Fun}

We used Tax4Fun of predicted metagenome content to impute the metagenome from our 16S rRNA sequencing results. We found that chronic noise exposure significantly increased KEGG orthologs (KOs) of sphingolipid metabolism (Level 3 KOs, Fig. 3A) and K01190 and K00602 (Level K, Fig. 3B) in WT mice, while K02035 significantly decreased after noise exposure (Level K, Fig. 3B). Tax4Fun assignment to Level 3 and Level K KOs suggested that the most predicted functional categories in KEGG pathways were significantly altered after noise exposure in APP/PS1 mice, including increased KOs of DNA replication proteins and glycerophospholipid metabolism (Level 3 KOs, Fig. 3C), K1190, K1810, K1892, and K1755 (Level K, Fig. 3D). These results implied that chronic noise changed phospholipid and galactose metabolism and were relevant to oxidative status as well as the occurrence and development of EOAD pathology.

Chronic noise exposure affects oxidative and inflammatory responses in the intestine of WT and APP/PS1 mice

To determine whether dysfunction of the gut-brain axis caused by intestinal flora dysbiosis after noise exposure was mediated by oxidative dysfunction, the gut homeostasis of the oxidation and anti-oxidation system was investigated. The activities of T-AOC, GST, CAT, and GSH-Px were significantly decreased in noise-exposed mice in both the WT and APP/PS1 groups, whereas the noise-induced downregulation of Nrf2 only occurred in APP/PS1 groups. APP/PS1 overexpression significantly decreased the activities of GST and T-AOC, with a trend towards decreasing activities of CAT and GSH-Px (Fig. 4A-D, L; ANOVA output: T-AOC: exposure $F(1,18)=20.49, P=0.0002$, genotype $F(1,18)=34.54, P<0.0001$, interaction $F(1,18)=0.158, P=0.6955 ;$ CAT: exposure $F(1,18)=24.53, P=0.0001$, genotype $F(1,18)=0.2323, P=$ 0.6356 , interaction $F(1,18)=0.3593, P=0.5564$; GST: exposure $F(1,18)=10.47, P=0.0044$, genotype $F(1,18)=6.779, P=0.0175$, interaction $F(1,18)=0.5839, P=0.4542$; $G S H-P x$ : exposure $F(1,18)=14.63, P=$ 0.0012 , genotype $F(1,18)=4.286, P=0.0531$, interaction $F(1,18)=0.9393, P=0.3453$; Nrf2: exposure 
$F(1,17)=7.453, P=0.0143$, genotype $F(1,17)=7.501, P=0.0140$, interaction $F(1,17)=0.566, P=0.0762$ ). Expression levels of GCLC, GSR, GSS, and GSTpi were affected by chronic noise exposure in both WT and APP/PS1 groups. APP/PS1 overexpression significantly reduced expression levels of GSS and GSTpi (Fig. 4F-I, ANOVA output: GCLC: exposure $F(1,20)=62.13, P<0.0001$, genotype $F(1,20)=3.738, P=0.0675$, interaction $F(1,20)=0.8248, P=0.3746$; GSR: exposure $F(1,20)=135.3, P<0.0001$, genotype $F(1,20)=$ 8.739, $P=0.0078$, interaction $F(1,18)=0.07271, P=0.7902$; GSS: exposure $F(1,20)=344.4, P<0.0001$, genotype $F(1,20)=210.3, P<0.0001$, interaction $F(1,20)=59.87, P<0.0001$; GSTpi: exposure $F(1,20)=$ $28.14, P<0.0001$, genotype $F(1,20)=19.7, P=0.0003$, interaction $F(1,20)=1.404, P=0.2499)$. Next to antioxidant capacity, the activity of the mitochondrial enzyme SDH was decreased markedly after noise exposure in both WT and APP/PS1 mice (Fig. 4E, exposure $F(1,18)=42.19, P<0.0001$, genotype $F(1,18)=$ 31.86, $P<0.0001$, interaction $F(1,18)=0.8051, P=0.3814)$, while the expression of the NOX2 and NOX4, the main resource of reactive oxygen species (ROS), was upregulated by noise exposure in APP/PS1 mice (Fig. 4J, K, NOX2: exposure $F(1,20)=8.443, P=0.0087$, genotype $F(1,20)=14.11, P=0.0012$, interaction $F(1,20)=0.09169, P=0.7652$; NOX4: exposure $F(1,20)=8.076, P=0.0101$, genotype $F(1,20)=10.65, P=$ 0.0039 , interaction $F(1,20)=2.6, P=0.1225)$, which may contribute to the progression of dysfunction of aerobic oxidative respiratory chain.

Oxidative stress and inflammatory responses reciprocally interact in a causal manner. We, thus, assessed the levels of gut inflammatory-associated mediators. The levels of IL-6, NF-KB, iNOS, and NGAL were significantly altered or showed a trend towards being altered by chronic noise and APP/PS1 overexpression (Fig. 5A-D, ANOVA output: IL-6: exposure $F(1,18)=24.27, P=0.0001$, genotype $F(1,18)=$ $0.5591, P=0.4643$, interaction $F(1,18)=0.005064, P=0.9441$; NF-KB: exposure $F(1,18)=16.77, P=$ 0.0007 , genotype $F(1,18)=1.862 E-5, P=0.9966$, interaction $F(1,18)=0.02503, P=0.8760$; iNOS: exposure $F(1,18)=14.11, P=0.0014$, genotype $F(1,18)=0.3083, P=0.5855$, interaction $F(1,18)=0.0132, P=0.9098$; NGAL: exposure $F(1,18)=19.94, P=0.0003$, genotype $F(1,18)=1.268, P=0.2758$, interaction $F(1,18)=$ $0.8862, P=0.3597)$.

Chronic noise-induced impairment of tight junctions in the intestine and hippocampus of WT and APP/PS1 mice

To determine the status of intestinal and blood-brain barrier (BBB) permeability, we evaluated the expression levels of main tight junction proteins by using western blot analysis. Immunoblotting confirmed reduced intestinal expression of CLDN1, occludin, and ZO-1 in WT and APP/PS1 transgenic mice following noise exposure. APP/PS1 overexpression tended to decrease the levels of epithelial tight junction proteins (Fig. 6A-D, ANOVA output: CLDN1: exposure $F(1,16)=49.61, P<0.0001$, genotype $F(1,16)=10.91, P=0.0045$, interaction $F(1,16)=0.001969, P=0.9652 ;$ occludin: exposure $F(1,16)=18.01$, $P=0.0006$, genotype $F(1,16)=16.83, P=0.0008$, interaction $F(1,16)=0.02282, P=0.8818$; ZO-1: exposure $F(1,16)=39.39, P<0.0001$, genotype $F(1,16)=10.29, P=0.0055$, interaction $F(1,16)=0.4828, P=0.4971)$. Similar expression patterns of CLDN1, occludin, and ZO-1 were observed in the hippocampus of noiseexposed APP/PS1 mice. The expression of occludin in the hippocampus of noise-exposed WT mice was significantly reduced, while CLDN1 and ZO-1 expression only showed a trend to decrease without 
reaching significance (Fig. 6E-H, ANOVA output: CLDN1: exposure $F(1,16)=14.61, P=0.0015$, genotype $F(1,16)=5.796, P=0.0285$, interaction $F(1,16)=0.1203, P=0.7332$; occludin: exposure $F(1,16)=26.08, P$ $=0.0001$, genotype $F(1,16)=9.861, P=0.0063$, interaction $F(1,16)=0.1906, P=0.6682 ; Z O-1$ : exposure $F(1,16)=29.46, P<0.0001$, genotype $F(1,16)=29.05, P<0.0001$, interaction $F(1,16)=2.201, P=0.1573)$. These data suggested that chronic noise-induced impairments in the epithelial integrity of the intestine and BBB open the gut brain axis pathway of pathological substances, which may be synergistically aggravated by APP/PS1 overexpression.

\section{Abnormalities in neurotransmitters and inflammatory responses following noise exposure}

To further explore the flora dysbiosis-triggered pathology response in the gut-brain axis, we examined serum levels of neurotransmitters (5-HT and GABA) and inflammatory mediators (D-LAC, endotoxin, IL-6, and TNF) using ELISAs. Noise exposure significantly decreased 5-HT concentrations in blood in both WT and APP/PS1 mice. Noise-induced decreases in serum GABA levels were only observed in APP/PS1 mice, but no significant difference was observed (Fig. 7A and B, ANOVA output: $5-H T$ : exposure $F(1,22)=28.04$, $P<0.0001$, genotype $F(1,23)=6.009, P=0.0226$, interaction $F(1,23)=1.009, P=0.3261$; GABA: exposure $F(1,23)=0.8455, P=0.3683$, genotype $F(1,23)=1.844, P=0.1889$, interaction $F(1,23)=2.366, P=0.1389)$. Additionally, significantly elevated serum levels of endotoxin, IL-6, and TNF were observed in noiseexposed APP/PS1 mice (Fig. 7D-F). The expression of IL-6 in noise-exposed WT mice was significantly increased, while endotoxin and TNF levels only tended to increase, but this was not statistically significant (Fig. 7D-F, ANOVA output:endotoxin: exposure $F(1,23)=17.56, P=0.0004$, genotype $F(1,23)=$ $0.285, P=0.5986$, interaction $F(1,23)=0.7259, P=0.4030$; IL6: exposure $F(1,23)=25.14, P<0.0001$, genotype $F(1,23)=12.64, P=0.0018$, interaction $F(1,23)=0.3546, P=0.5576$; TNF: exposure $F(1,23)=$ $9.067, P=0.0062$, genotype $F(1,23)=32.96, P<0.0001$, interaction $F(1,23)=4.145, P=0.0534)$. In addition, noise exposure tended to increase serum D-LAC concentration in WT and APP/PS1 mice, but this did not reach significance (Fig. 7C, ANOVA output: exposure $F(1,20)=8.642, P=0.0081$, genotype $F(1,20)=3.043, P=0.0964$, interaction $F(1,20)=0.01405, P=0.9068)$. These results suggested that chronic noise may affect gut microbiota-related neurochemistry and systemic inflammation, potentially contributing to the accumulation of AD-related pathology.

\section{Noise exposure induces AD-like neuropathology in the hippocampus of WT and APP/PS1 mice}

To assess the effects of the CNS end of the gut-brain axis following chronic noise exposure, we examined levels of $A \beta 40$ and $A \beta 42$ using ELISA and relative expression of phosphorylated tau at $A D$ related sites by western blot analysis in hippocampal tissues. The hippocampal content of $A \beta 40$ and $A \beta 42$ was significantly elevated in the noise-exposed groups compared to those in the corresponding WT or APP/PS1 control groups (Fig. 8A and B, ANOVA output: $A \beta 40$ : exposure $F(1,20)=49.57, P<0.0001$, genotype $F(1,20)=44.26, P<0.0001$, interaction $F(1,20)=7.396, P=0.0132 ; A \beta 42$ : exposure $F(1,20)=$ 
83.86, $P<0.0001$, genotype $F(1,20)=130.1, P<0.0001$, interaction $F(1,20)=23.29, P=0.0001)$. Tau phosphorylation at Ser404, Ser202, and Thr205 sites was significantly higher in the noise-exposed groups than in the WT or APP/PS1 control groups (Fig. 8C-E, ANOVA output: Ser404: exposure F(1,16) $=112.5, P$ $<0.0001$, genotype $F(1,16)=0.05214, P=0.8223$, interaction $F(1,16)=15.34, P=0.0012$; AT8: exposure $F(1,16)=17.36, P=0.0007$, genotype $F(1,16)=1.345, P=0.2632$, interaction $F(1,16)=0.004014, P=$ 0.9503). Neither exposure nor genotype affected protein expression of non-phosphorylated tau (Tau1) (Fig. 7F, ANOVA output: Tau1: exposure $F(1,16)=0.006968, P=0.9345$, genotype $F(1,16)=0.1539, P=$ 0.7001 , interaction $F(1,16)=0.00006, P=0.9941)$. These data suggested that chronic noise exposure and APP/PS1 genotype aggravated AD-related pathological alterations in a synergistic manner.

\section{Discussion}

In this study, we confirmed that chronic noise can cause a series of microbiome-gut-brain axis events in the EOAD transgenic mice model, including abnormalities in the composition of gut microbial community and oxidative-inflammatory state, disrupted tight junction function in the intestine and BBB, and systemic inflammatory responses associated with amyloid pathology and tau hyperphosphorylation. Moreover, we found evidence that combined exposure to environmental noise stress and APP/PS1 overexpression may synergistically result in a systemic oxidative-inflammatory status driven by shifts in microbial load and metabolism, which may add a new dimension to certain biological markers of AD pathology to facilitate the onset or progression of EOAD.

The composition of intestinal flora is easily influenced by environmental factors [25], which is a susceptible event that drives subsequent gut-brain axis effects. Our results demonstrated that environmental noise exposure not only aggravated abnormalities in the gut microbiota composition but also significantly decreased the abundance of Rikenellaceae and Ruminococcaceae families as well as that of Anaerovorax, Lachnospira, and Odoribacter genus in microbial communities, particularly in the APP/PS1 overexpression groups. Moreover, chronic noise exposure increased the abundance of Staphylococcus, a conditional pathogen that has previously been associated with elevated endotoxemia [26], which is known to increase inflammatory cytokines, cause endothelial dysfunction in humans, and to be associated with metabolic syndrome [27]. The abundance of Rikenellaceae was negatively correlated with metabolism and cardiovascular diseases [28]. Notably, other bacteria with significant changes in abundance belong to butyrate-producing groups [29,30]. Interestingly, a typical feature of intestinal microflora imbalance is a decrease in the abundance of common butyrate-producing bacteria and an increase in the number of conditional pathogens [31]. Butyrate is an energy source for epithelial cells and can protect cells from oxidative stress; it maintains redox homeostasis and mitochondrial energy metabolism through a regulation of the GSK-3ß/Nrf2 pathway [32]. A human study further demonstrated that colonic administration of butyrate mainly resulted in an increased transcriptional regulation of the pathways representing fatty acid oxidation, electron transport chain, and oxidative stress [33]. Moreover, butyrate intervention has an effect on inflammation [32], epithelial integrity, and apoptosis [33]. The present findings suggest that intestinal flora dysbiosis-related oxidative damage may 
play a key role in the subsequent gut-brain axis effects induced by noise exposure, which may have important etiological implications.

We observe that that the dysfunction in KEGG pathways related to the metabolism of phospholipid and galactose in functional predictions via Tax4Fun is consistent with the findings of a previous study showing that metabolic mechanisms of stress-related cognitive impairments in APP/PS1 mice were partly related to sphingolipid metabolism [35]. Abnormal phospholipid metabolism is closely related to oxidative stress and inflammatory responses and plays a significant role in the occurrence and development of cardiovascular and cerebrovascular diseases [36]. Accumulation of D-galactose triggers ROS generation and leads to oxidative stress and inflammation, which is widely used to mimic aging in animal models [37]. A comprehensive analysis of the aforementioned results of flora composition and functional prediction highlight pathways corresponding to oxidative stress and the development of cell senescence, although the Tax4Fun prediction analysis has its limitations in accuracy and the underlying mechanisms need further elucidation. If true, this hypothesis would imply that systemic inflammatory responses and subsequent gut-brain axis disruption by environmental noise could be mediated by microbiota dysbiosis involving oxidation-inflammation pathways.

Recent researches have demonstrated that gut microbiota shift toward proinflammatory bacteria in APP/PS1 transgenic mice [38], and influence mitochondrial function by regulating ROS production [39, 40], suggesting that the intestinal microbiota-regulated homeostasis of oxidation-inflammation plays key roles in EOAD pathogenesis [41]. The cellular mechanism of ROS overproduction is closely related to mitochondrial dysfunction, which is promoted by defective electron transport chain enzymes (i.e., SDH and NOX) and inactivation of antioxidant mitochondrial enzymes (i.e., GSH, GST, and CAT) [42, 43]. Moreover, Nrf2-related signaling is a key factor in oxidative stress, which regulates the enzymes involved in antioxidative stress responses by interacting with antioxidant response elements and underlies cellular repair mechanisms in inflammatory states [44]. There exists a bidirectional relationship between oxidative stress and inflammation; oxidative impairment can be induced by inflammatory responses, and inflammation can be triggered or aggravated by ROS through activation of NF-KB, which controls the expression of genes involved in inflammatory responses [45]. Hence, altered microbial populations may dysregulate redox balance and consequently cause inflammatory responses. Our previous data demonstrated systemic inflammatory responses $[21,24]$ and reciprocal activation of pro-inflammatory cytokines and astrocytes following noise exposure, which could cause a positive feedback loop, resulting in continuous accumulation of low-level neuropathology [12]. In this study, we confirmed that environmental noise or APP/PS1 overexpression increased intestinal oxidative stress via dysregulation of Nox2/4 and antioxidant-related enzymes, which was similar to recent researches $[17,18]$. In either scenario, noise-induced abnormalities, including oxidative stress, systemic inflammation, and a trend for increases in serum D-LAC, were consistent with the results of flora composition and functional prediction, again suggesting that the dysbiosis of intestinal flora initiates gut-brain axis effects caused by noise exposure. Importantly, a recent study demonstrated that Nrf2 deficiency impairs barrier integrity by disrupting the energy-dependent tight junction [46]. Thus, it is reasonable to postulate that noise-induced 
Nrf2-related antioxidative dysfunction may also contribute to tight junction pathology in the gut-brain axis.

The present study revealed that both chronic noise exposure and APP/PS1 overexpression caused dysregulation of the expression of tight junction proteins, including claudin 1, occludin, and Z0-1, in the intestine and hippocampus, demonstrating widespread deficiency in intestinal barrier function and BBB integrity. The epithelial tight junction transmembrane proteins (claudins and occludin) and cytoplasmic membrane proteins (ZO-1 and ZO-2) maintain the permeability function of the BBB and intestinal barrier [47-49], which can be downregulated by oxidative stress on inhibition of CaMKKB-AMPK signaling[50]. Moreover, oxidative stress can directly affect permeability and influence homeostasis of the intestinal epithelial barrier, thus increasing the risk of intestinal flora reaching the circulatory system and CNS through the gut-brain axis [51]. Thus, these results at least partly explain the elevated serum levels of xenobiotic molecules and inflammatory mediators, which play a major role in the low-grade systemic inflammation of the gut-brain axis and neurochemical dysregulation underscoring neurodegeneration.

Gut microbiota dysbiosis and its induction of oxidative and inflammatory imbalance triggers a cascade response in the gut-brain axis due to BBB disruption and aggravates AD-like cerebral pathology via immune, metabolite-mediated, and oxidative signaling pathways [52]. A recent human brain study suggested that loss of cortical tight junction proteins occurs in association with the accumulation of ADrelated insoluble proteins, particularly $A B$ [53]. Moreover, it is noteworthy that the loss of tight junction proteins was associated with a loss of synaptic markers [53], which play key roles in cognitive decline in $A D$. Since $A D$ is characterized by the progressive accumulation of insoluble proteins and cognitive deterioration, it is reasonable to postulate that tight junction dysfunction in the gut-brain axis may contribute to the onset or/and progression of AD-like neuropathology. Our results indicate that the presence of dysbiosis and oxidative stress, causing the breakdown of the intestinal and BBB permeability and systemic spread of inflammatory condition, accelerated $A \beta$ production and tau hyperphosphorylation in a synergistic manner with APP/PS1 overexpression. The downregulation of serum 5-HT and GABA in noise-exposed mice closely associated with gut microbiota disruption and enhanced hippocampal $A \beta$ synthesis provide further evidence for the microbiome-gut-brain axis underlying the mechanism of $A D$ development. Additionally, we demonstrated that 30 days of exposure of environmental noise was sufficient to facilitate the occurrence and development of AD-like pathologies in 3-month-old APP/PS1 Tg mice, which have an accelerated EOAD phenotype characterized by elevated $A \beta$ deposits at an age as young as $13-16$ weeks old [22].

The mutations in PSEN1, PSEN2, or APP genes can only explain the pathogenesis of a small number of cases, and their precise contribution is not fully clarified. Environmental stress increased glucocorticoid levels, AD-like neuropathology, and cognitive impairments in APP/PS1 mice, implying that EOAD model mice are more vulnerable to environmental stress than WT mice [15]. This line of thinking also raises the possibility that the synergy between environmental noise and APP/PS1 overexpression may underlie the pathogenesis of EOAD. To our knowledge, there is scant epidemiological evidence for the relationship 
between environmental stressor exposure and EOAD development. Experimental data based on the APP/PS1 EOAD model, including data from this study, may provide important clues in this regard.

\section{Conclusions}

Taken together, exposure to long-term environmental noise, likely via the associated alterations in microbiome-gut-brain axis status, can exacerbate amyloid and tau pathology in early-aged EOAD model mice. Noise exposure upregulates oxidative stress and systemic low-grade inflammation, which may underlie epithelial barrier deficits in the intestine and brain, and may thus be an environmental risk factor for EOAD in vulnerable individuals. These findings enhance our understanding of the etiological signaling pathways involved in EOAD pathology following environmental hazard stress. Further, they suggest that low-grade oxidative stress and inflammatory responses in the gut-brain axis are potential biochemical and pathological markers in the presymptomatic stage of EOAD. Early identification may thus help to protect against the detrimental effects of chronic noise exposure.

\section{Abbreviations}

$A D$, Alzheimer's disease; EOAD, early-onset Alzheimer's disease; $A \beta$, amyloid- $\beta$; PSEN1, presenilin 1; PSEN2, presenilin 2; APP, amyloid precursor protein; APP/PS1, B6C3-Tg (APPswe, PSEN1dE9)/NJU; WT, C57BL/6Nju; SPL, sound pressure level; OTU, operational taxonomic unit; LEFSE, linear discriminant analysis LAD coupled with effect size measurements; T-AOC, total antioxidant capacity, CAT, catalase; GST, glutathione S-transferase; GSH-Px, glutathione peroxidase; Ct, threshold cycle; ELISA, enzyme-linked immunosorbent assay; GADPH, glyceraldehyde phosphate dehydrogenase; KO, KEGG ortholog; BBB, blood-brain barrier; SAMP8, senescence accelerated prone; NF-KB, nuclear factor-kappa B

\section{Declarations}

\section{Ethics approval and consent to participate}

All animal experiments were conducted according to the KU Leuven ethical guidelines and approved by the KU Leuven Committee on Animal Care.

\section{Consent for publication}

Not applicable.

\section{Availability of supporting data}

Raw data is available from the corresponding authors upon reasonable request.

\section{Competing interest}


None of the authors have any financial relationship with the organizations that sponsored the research. The other authors declare that they have no competing interests.

\section{Funding}

This work was supported by National Natural Science Foundation of China (No. 81673136) and Tianjin Natural Science Foundation (No. 17JCZDJC34900) awarded to B.C.

\section{Author contributions}

B.C. designed the research; H.C., W.C. and D.S., performed the exposure and behavior experiments; H.C., W.C., H.Y., X.S., X.G., K.W. K.M. and Z.L. performed the molecular biology experiments; B.C., H.C., W.C. and Z.L. analyzed data; B.C. wrote the paper. All authors read and approved the final manuscript.

\section{Acknowledgements}

Not applicable.

\section{Author information}

${ }^{1}$ Tianjin Institute of Environmental and Operational Medicine, Tianjin, China. ${ }^{2}$ School of Public Health and Management, Weifang Medical University, Weifang, China. ${ }^{3}$ College of Public Health, North China University of Science and Technology, Tangshan, China. ${ }^{4}$ Zibo Center for Disease Control and Prevention, Zibo, China.

\section{References}

1. K. Blennow, M.J. de Leon, H. Zetterberg, Alzheimer's disease, Lancet 368 (9533) (2006) 387-403. 2. S. Gandy, Perspective: prevention is better than cure, Nature 475 (7355) (2011) S15. 3. D. Campion, C. Dumanchin, D. Hannequin, B. Dubois, S. Belliard, M. Puel, C. Thomas-Anterion, A. Michon, C. Martin, F. Charbonnier, G. Raux, A. Camuzat, C. Penet, V. Mesnage, M. Martinez, F. Clerget-Darpoux, A. Brice, T. Frebourg, Early-onset autosomal dominant Alzheimer disease: Prevalence, genetic heterogeneity, and mutation spectrum, Am. J. Hum. Genet. 65(3) (1999) 664-670. 4. L. Hoeijmakers, S.R. Ruigrok, A. Amelianchik, D. Ivan, A.M. van Dam, P.J. Lucassen, A. Korosi, Early-life stress lastingly alters the neuroinflammatory response to amyloid pathology in an Alzheimer's disease mouse model, Brain Behav Immun. 63 (2017) 160-175. 5. O. Hahad, J.H. Prochaska, A. Daiber, T. Muenzel, Environmental noiseinduced effects on Stress Hormones, Oxidative stress, and vascular dysfunction: Key factors in the relationship between cerebrocardiovascular and psychological disorders, Oxid. Med. Cell. Longev. 2019(2019):4623109. 6. D. Hjortebjerg, A.M. Andersen, J.S. Christensen, M. Ketzel, O. Raaschou-Nielsen, J. Sunyer,J. Julvez, J. Forns, M. Sørensen, Exposure to road traffic noise and behavioral problems in 7year-old children: a cohort study, Environ. Health Perspect. 124 (2) (2016) 228-234. 7. B. Cui, M. Wu, X. She, Effects of chronic noise exposure on spatial learning and memory of rats in relation to neurotransmitters and NMDAR2B alteration in the hippocampus, J. Occup. Health 51(2) (2009) 152-158. 
8. G.Q. Di, Z.Q. Qin, Influences of combined traffic noise on the ability of learning and memory in mice. Noise Health 20 (92) (2018) 9-15. 9. L. Cheng, S.H. Wang, Q.C. Chen, X.M. Liao, Moderate noise induced cognition impairment of mice and its underlying mechanisms, Physiol. Behav. 104 (5) (2011) 981-988. 10. B. Cui, L. Zhu, X. She, M. Wu, Q. Ma, T. Wang, H. Liu, Chronic noise exposure causes persistence of tau hyperphosphorylation and formation of NFT tau in the rat hippocampus and prefrontal cortex, Exp. Neurol. 238 (2) (2012) 122-129. 11. B. Cui, M.Q. Wu, L. Zhu, X. She, Q. Ma, H. Liu, Effect of chronic noise exposure on expression of N-methyl-D-aspartic acid receptor 2B and Tau phosphorylation in hippocampus of rats, Biomed. Environ. Sci. 26 (3) (2013) 163-168. 12. B. Cui, K. Li, Z. Gai, X. She, N. Zhang, C. Xu, X. Chen, G. An, Q. Ma, R. Wang, Chronic Noise exposure acts cumulatively to exacerbate Alzheimer's disease-like amyloid- $\beta$ pathology and neuroinflammation in the rat hippocampus, Sci. Rep. 5 (2015) 12943. 13. S. Manikandan, M.K. Padma, R. Srikumar, N. Jeya Parthasarathy, A. Muthuvel, R. Sheela Devi, Effects of chronic noise stress on spatial memory of rats in relation to neuronal dendritic alteration and free radical-imbalance in hippocampus and medial prefrontal cortex, Neurosci. Lett. 399 (12) (2006) 17-22. 14. W. Li, D. Su, Q. Zhai, H. Chi, X. She, X. Gao, K. Wang, H. Yang, R. Wang, B. Cui, Proteomes analysis reveals the involvement of autophagy in $A D$-like neuropathology induced by noise exposure and ApoE4, Environ. Res. 176 (2019) 108537. 15. B. Han, L. Yu, Y. Geng, L. Shen, H. Wang, Y. Wang, J. Wang, M. Wang, Chronic stress aggravates cognitive impairment and suppresses insulin associated signaling pathway in APP/PS1 mice, J. Alzheimers Dis. 53(4)(2016)1539-1552. 16. J. Herzog, F.P. Schmidt, O. Hahad, S.H. Mahmoudpour, A.K. Mangold, P. Garcia Andreo, J. Prochaska, T. Koeck, P.S. Wild, M. Sørensen, A. Daiber,T. Münzel, Acute exposure to nocturnal train noise induces endothelial dysfunction and pro-thromboinflammatory changes of the plasma proteome in healthy subjects, Basic Res. Cardiol. 114(6) (2019) 46. 17. T. Münzel, A. Daiber, S. Steven, L.P. Tran, E. Ullmann, S. Kossmann, F.P. Schmidt, M. Oelze 1, N. Xia, H. Li , A. Pinto, P. Wild, K. Pies, E.R. Schmidt, S. Rapp, S. Kröller-Schön, Effects of noise on vascular function, oxidative stress, and inflammation: mechanistic insight from studies in mice, Eur. Heart J. 38(37)( 2017) 2838-2849. 18. S. Kröller-Schön, A. Daiber, S. Steven, M. Oelze, K. Frenis, S. Kalinovic, A. Heimann, F.P. Schmidt, A. Pinto, M. Kvandova, K. Vujacic-Mirski, K. Filippou, M. Dudek, M. Bosmann, M. Klein, T. Bopp, O. Hahad, P.S. Wild, K. Frauenknecht, A. Methner, .E.R Schmidt, S. Rapp, H. Mollnau, T. Münzel, Crucial role for Nox2 and sleep deprivation in aircraft noise-induced vascular and cerebral oxidative stress, inflammation, and gene regulation, Eur. Heart. J. 9(38) (2018)3528-3539. 19. R. M. Jones, J. W. Mercante, A. S. Neish, Reactive oxygen production induced by the gut microbiota: pharmacotherapeutic implications, Curr. Med. Chem.19(10) (2012) 1519-1529. 20. L. Dumitrescu, I. Popescu-Olaru, L. Cozma, D. Tulbă, M.E. Hinescu, L.C. Ceafalan, M. Gherghiceanu, B.O. Popescu, Oxidative stress and the microbiota-gut-brain axis, Oxid. Med. Cell. Longev. 2018 (2018) 2406594. 21. B. Cui, Z. Gai, X. She, R. Wang, Z. Xi, Effects of chronic noise on glucose metabolism and gut microbiotahost inflammatory homeostasis in rats, Sci. Rep. 6 (2016) 36693. 22. L. Holcomb, M.N. Gordon, E. Mcgowan, X. Yu, S. Benkovic, P. Jantzen, K. Wright, I. Saad, R. Mueller, D. Morgan, S. Sanders, C. Zehr, K. O'Campo, J. Hardy, C.M. Prada, C. Eckman, S. Younkin, K. Hsiao, K. Duff, Accelerated Alzheimer-type phenotype in transgenic mice carrying both mutant amyloid precursor protein and presenilin 1 transgenes, Nat. Med. 4 (1998) 97-100. 23. D. Laukens, B.M. Brinkman,J. Raes, M. De Vos, P. Vandenabeele, Heterogeneity of the gut microbiome in mice: guidelines for optimizing experimental 
design, F.E.M.S. Microbiol. Rev. 40 (2016) 117-132. 24. B. Cui, D. Su, W. Li, X. She, M. Zhang, R. Wang, Q. Zhai, Effects of chronic noise exposure on the microbiome-gut-brain axis in senescence-acceleratedprone mice: implications for Alzheimer's disease, J. Neuroinflammation 15 (2018) 190. 25. D. Rothschild, O. Weissbrod, E. Barkan, A. Kurilshikov, T. Korem, D. Zeevi, P.I. Costea, A. Godneva, I. N. Kalka, N. Bar, S. Shilo, D. Lador, A.V. Vila, N. Zmora, M. Pevsner-Fischer, D. Israeli, N. Kosower, G. Malka, B.C. Wolf, T. Avnit-Sagi, M. Lotan-Pompan, A. Weinberger, Z. Halpern, S. Carmi, J. Fu, C. Wijmenga, A. Zhernakova, E. Elinav, E. Segal, Environment dominates over host genetics in haping human gut microbiota, Nature 555 (2018) 210-215. 26. Y. Nakamura, J. Oscherwitz, K.B. Cease, S.M. Chan, R. Muñoz-Planillo, M. Hasegawa, A.E.

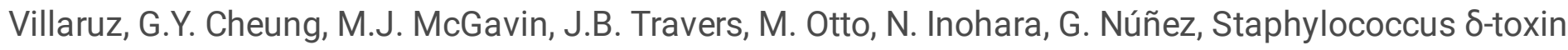
induces allergic skin disease by activating mast cells, Nature 503 (2013) 397-401. 27. N.E. Boutagy, R.P. McMillan, M.I. Frisard, M.W. Hulver, Metabolic endotoxemia with obesity: is it real and is it relevant? Biochimie. 124 (2016) 11-20. 28. S.F. Clarke, E.F. Murphy, O. O'Sullivan, R.P. Ross, P.W. O'Toole, F. Shanahan, P.D. Cotter, Targeting the microbiota to address diet-induced obesity: a time dependent challenge, PLoS One 8 (2013) e65790. 29. C. Matthies, S. Evers, W. Ludwig, B. Schink, Anaerovorax odorimutans gen. nov., sp. nov., a putrescine-fermenting, strictly anaerobic bacterium, Int. J. Syst Evol Microbiol. 50 (2000) 1591-1594. 30. C. Menni, C. Lin, M. Cecelja, M. Mangino, M.L. Matey-Hernandez, L. Keehn, R.P. Mohney, C.J. Steves, T.D. Spector, C.F. Kuo, P. Chowienczyk, A.M. Valdes, Gut microbial diversity is associated with lower arterial stiffness in women, Eur. Heart J. 39 (2018) 2390-2397. 31. J. Qin, Y. Li, Z. Cai, S. Li, J. Zhu, F. Zhang, S. Liang, W. Zhang, Y. Guan, D. Shen, Y. Peng, D. Zhang, Z. Jie, W. Wu, Y. Qin, W. Xue, J. Li, L. Han, D. Lu, P. Wu, Y. Dai, X. Sun, Z. Li, A. Tang, S. Zhong, X. Li, W. Chen, R. Xu, M. Wang, Q. Feng, M. Gong, J. Yu, Y. Zhang, M. Zhang, T. Hansen, G. Sanchez, J. Raes, G. Falony, S. Okuda, M. Almeida, E. LeChatelier, P. Renault, N. Pons, J.M. Batto, Z. Zhang, H. Chen, R. Yang, W. Zheng, S. $\mathrm{Li}, \mathrm{H}$. Yang, J. Wang, S.D. Ehrlich, R. Nielsen, O. Pedersen, K. Kristiansen, J. Wang, A metagenome-wide association study of gut microbiota in type 2 diabetes, Nature 490 (2012): 55-60. 32. X. Xing, Z. Jiang, X. Tang, P. Wang, Y. Li, Y. Sun, G. Le, S. Zou, Sodium butyrate protects against oxidative stress in HepG2 cells through modulating Nrf2 pathway and mitochondrial function, J. Physiol. Biochem. 73(3) (2016) 405-414. 33. S.A. Vanhoutvin, F.J. Troost, H.M. Hamer, P.J. Lindsey, G.H. Koek, D.M. Jonkers,A. Kodde,K. Venema, R.J. Brummer, Butyrate-induced transcriptional changes in human colonic mucosa, PLoS One 4(8)(2009) e6759. 34. H.M. Hamer, D. Jonkers, K. Venema, S. Vanhoutvin, F.J. Troost,R.J. Brummer , Review article: the role of butyrate on colonic function, Aliment. Pharmacol. Ther. 27 (2008) 104-119. 35. B. Han, J.H. Wang, Y. Geng, L. Shen, H.L. Wang, Y.Y. Wang, M.W. Wang, Chronic Stress Contributes to Cognitive Dysfunction and Hippocampal Metabolic Abnormalities in APP/PS1 Mice, Cell. Physiol. Biochem. 41 (2017) 1766-1776. 36. C. López-Pedrera, N. Barbarroja, Y. Jimenez-Gomez, E. CollantesEstevez, M.A. Aguirre, M.J. Cuadrado, Oxidative stress in the pathogenesis of atherothrombosis associated with anti-phospholipidsyndrome and systemic lupus erythematosus: new therapeutic approaches, Rheumatology (Oxford) 55 (2016) 2096-2108. 37. W. Wu, C.L. Hou, X.P. Mu, C. Sun, Y.C. Zhu, M.J. Wang, Q.Z. Lv, H2S donor NaHS changes the production of endogenous H2S and NO in D-galactoseinduced accelerated ageing, Oxid. Med. Cell. Longev. 2017 (2017) 5707830. 38. C. Bäuerl, M.C. Collado, A. Diaz Cuevas, J. Viña, G. Pérez Martínez, Shifts in gut microbiota composition in an APP/ PSS1 transgenic mouse model of Alzheimer's disease during lifespan, Lett. Appl. Microbiol. 66 (6) (2018) 464-471. 39. W. 
Mottawea, C. K. Chiang, M. Mühlbauer, A.E. Starr, J. Butcher, T. Abujamel, S.A. Deeke, A. Brandel, H. Zhou, S. Shokralla, M. Hajibabaei, R. Singleton, E.I. Benchimol, C. Jobin, D.R. Mack, D. Figeys, A. Stintzi , Altered intestinal microbiota-host mitochondria crosstalk in new onset Crohn's disease, Nat. Commun. 7(1) (2016) 13419. 40. R.M. Jones, A.S. Neish, Redox signaling mediated by the gut microbiota, Free Radic. Biol. Med. 105 (2017) 41-47. 41. S. Xing, Y. Hu, X. Huang, D. Shen, C. Chen, Nicotinamide phosphoribosyltransferase-related signaling pathway in early Alzheimer's disease mouse models, Mol. Med. Rep. 20 (6) (2019) 5163-5171. 42. G. H. Kim, J. E. Kim, S. J. Rhie, S. Yoon, The role of oxidative stress in neurodegenerative diseases, Exp. Neurobiol. 24 (4) (2015) 325-340, . 43. J. Liu, L. Jia, J. Kan, C.H. Jin, In vitro and in vivo antioxidant activity of ethanolic extract of white button mushroom (Agaricus bisporus), Food Chem. Toxicol. 51 (2013) 310-316. 44. T.W. Kensler, N. Wakabayashi, S. Biswal. Cell survival responses to environmental stresses via the Keap1-Nrf2-ARE pathway. Annu. Rev. Pharmacol. Toxicol. 47(2007)89-116. 45. J. Leszek, G.E. Barreto, K.G. siorowski, E. Koutsouraki, M. Ávila-Rodrigues, G. Aliev, Inflammatory mechanisms and oxidative stress as key factors responsible for progression of neurodegeneration: role of brain innate immune system, C.N.S. Neurol. Disord. Drug Targets. 15 (2016) 329-336. 46. H. Chen, Y. Hu, Y. Fang, Z. Djukic, M. Yamamoto, N.J. Shaheen, R.C. Orlando, X. Chen, Nrf2 defificiency impairs the barrier function of mouse oesophageal epithelium, Gut 63(2014)711-719. 47. K. Spiegel, E. Tasali, R. Leproult, E. Van Cauter, Effects of poor and short sleep on glucose metabolism and obesity risk, Nat. Rev. Endocrinol. 5 (2009) 253-261. 48. E. Tasali, R. Leproult, D.A. Ehrmann, E. Van Cauter, Slow-wave sleep and the risk of type 2 diabetes in humans, Proc. Natl. Acad. Sci. U.S.A. 105 (2008) 1044-1049. 49. B.R. Stevenson, J.D. Siliciano, M.S. Mooseker, D.A. Goodenough, Identification of ZO-1: a high molecular weight polypeptide associated with the tight junction (zonula occludens) in a variety of epithelia, J. Cell Biol. 103 (1986) 755-766. 50. W.R. Yang, B.B. Li, Y. Hu, L. Zhang, X.Z. Wang, Oxidative stress mediates heat-induced changes of tight junction proteins in porcine sertoli cells via inhibiting CaMKKß-AMPK pathway, Theriogenology 142 (2020) 104-113. 51. A.T. Reese, E.H. Cho, B. Klitzman, S.P. Nichols, N.A. Wisniewski, M.M. Villa, H.K. Durand, S. Jiang, F.S. Midani, S.N. Nimmagadda, T.M. O'Connell, J.P. Wright, M.A. Deshusses, L.A. David, Antibiotic-induced changes in the microbiota disrupt redox dynamics in the gut, Elife 7 (2018) e35987. 52. A.M. Ton, C.M. Arpini, B.P. Campagnaro, T.M. Pereira, E.C. Vasquez, Alzheimer's disease: a brief update on the influence of gut microbiota and the impact of functional food, Food Microbiol. 2 (2018) 11-15. 53. Y. Yamazaki, M. Shinohara, M. Shinohara, A. Yamazaki, M.E. Murray, A.M. Liesinger, M.G. Heckman, E.R. Lesser, J.E. Parisi, R.C. Petersen, D.W. Dickson, T. Kanekiyo, G. Bu, Selective loss of cortical endothelial tight junction proteins during Alzheimer's disease progression, Brain 142(4) (2019) 1077-1092.

\section{Figures}


A
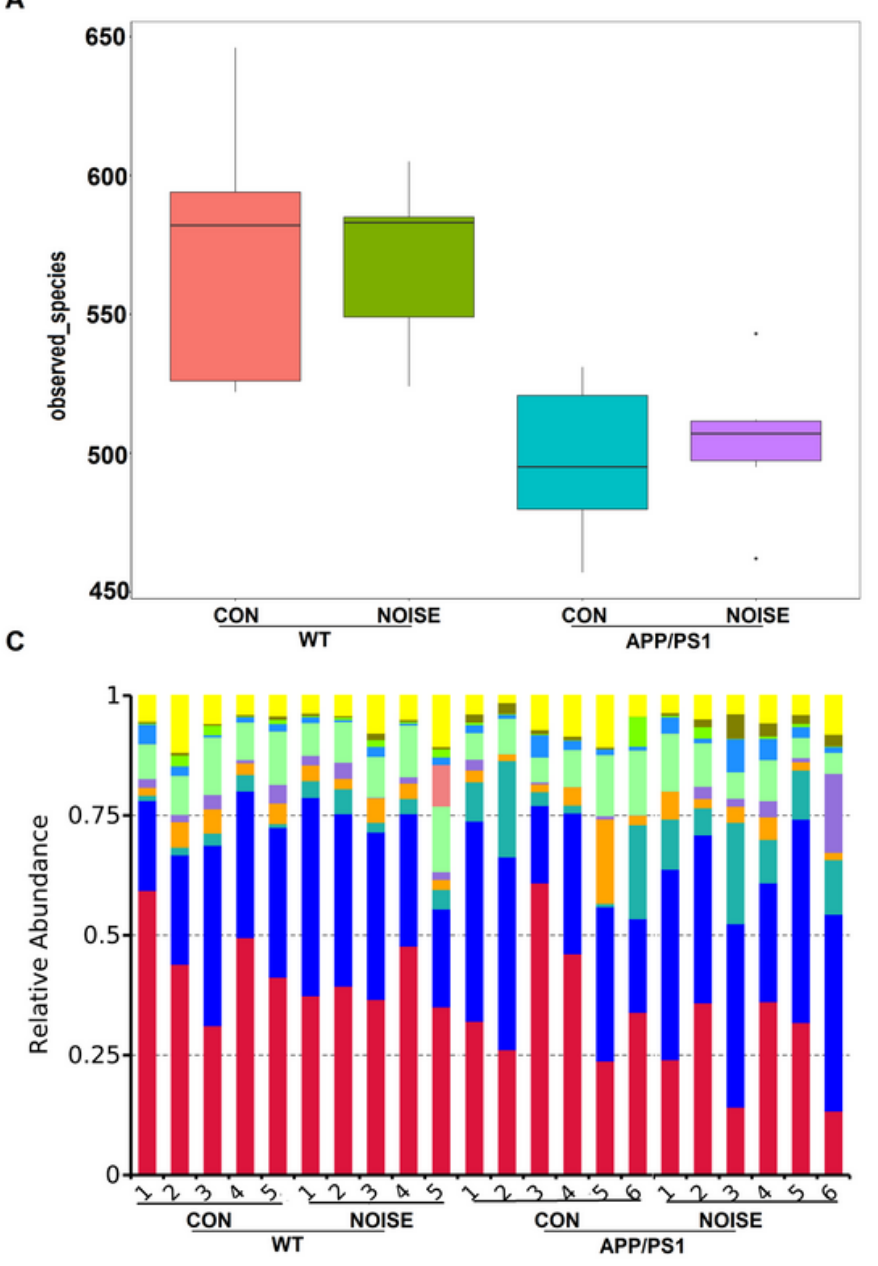

B

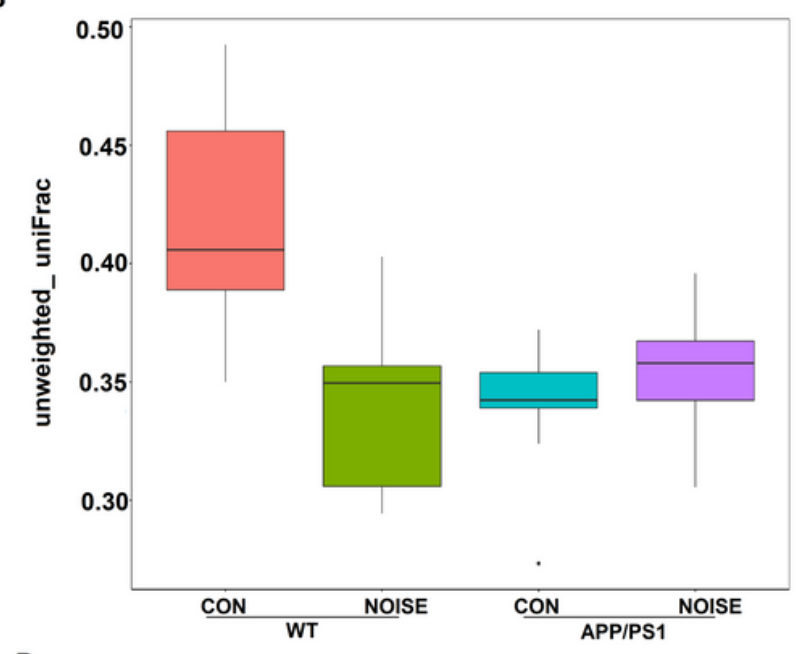

D

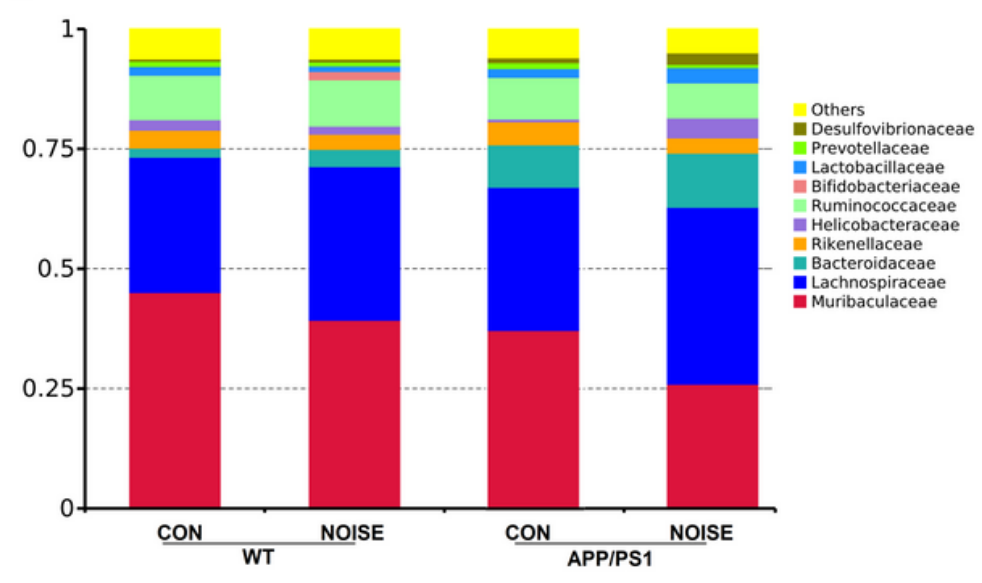

Figure 1

\section{Figure 1}

Summary of gut microbial communities in each group. (A) Alpha diversity of the microbial community in each group $(n=5-6)$. (B) Intragroup $\beta$-diversity of the microbial community in each group, as measured by unweighted UniFrac distance $(n=5-6)$. (C-D) Relative abundances of predominant bacteria at the family level in each sample and group ( $n=5-6)$. CON, control groups; NOISE, noise-exposed groups; WT, wild type mice; APP/PS1, APP/PS1 transgenic mice. 

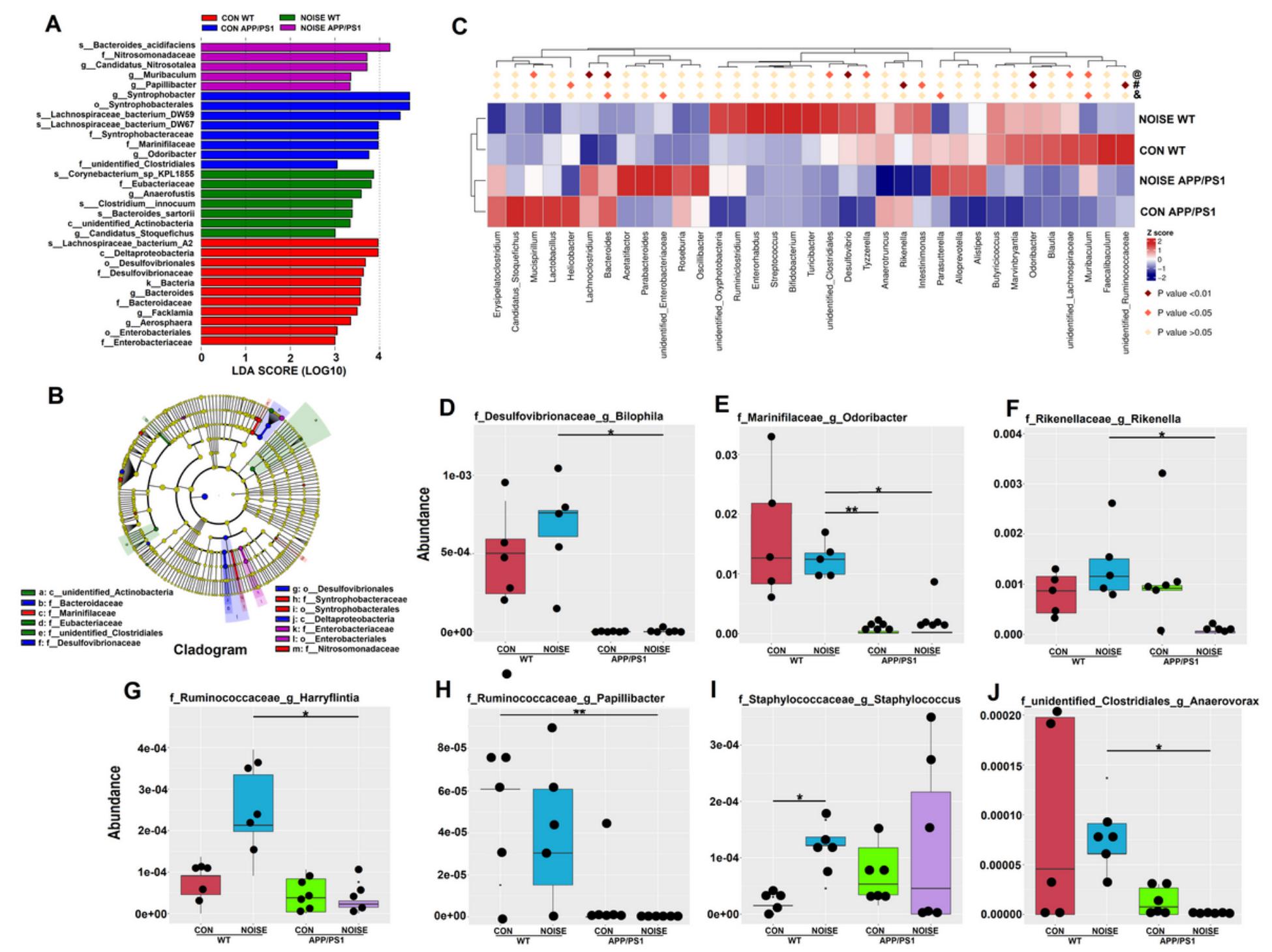

Figure 2

Figure 2

Alterations in gut microbiota composition following chronic noise exposure. (A) The most differentially abundant taxa in each group identified by LDA scores generated from the LEfSe analysis $(n=5-6)$. (B) The enriched taxa in fecal microbiota of mice are represented in cladograms. The central point represents the root of the tree (bacteria), and each ring represents the next lower taxonomic level (phylum to genus). The diameter of each circle represents the relative abundance of the taxon $(n=5-6)$. (C) A heatmap showing Metastat analysis of species with significant differences between groups based on species abundance tables at the bacterial genus level. (D-J) Comparison of relative abundance at the bacterial genus level in all groups. ${ }^{*} \mathrm{P}<0.05,{ }^{\star *} \mathrm{P}<0.01$, vs. indicated group, Mann-Whitney $\mathrm{U}$ test. CON, control groups; NOISE, noise-exposed groups; WT, wild type mice; APP/PS1, APP/PS1 transgenic mice; @, WT NOISE group vs. WT CON group; \#, APP/PS1 CON group vs. WT CON group; \&, APP/PS1 NOISE group vs WT CON group. 


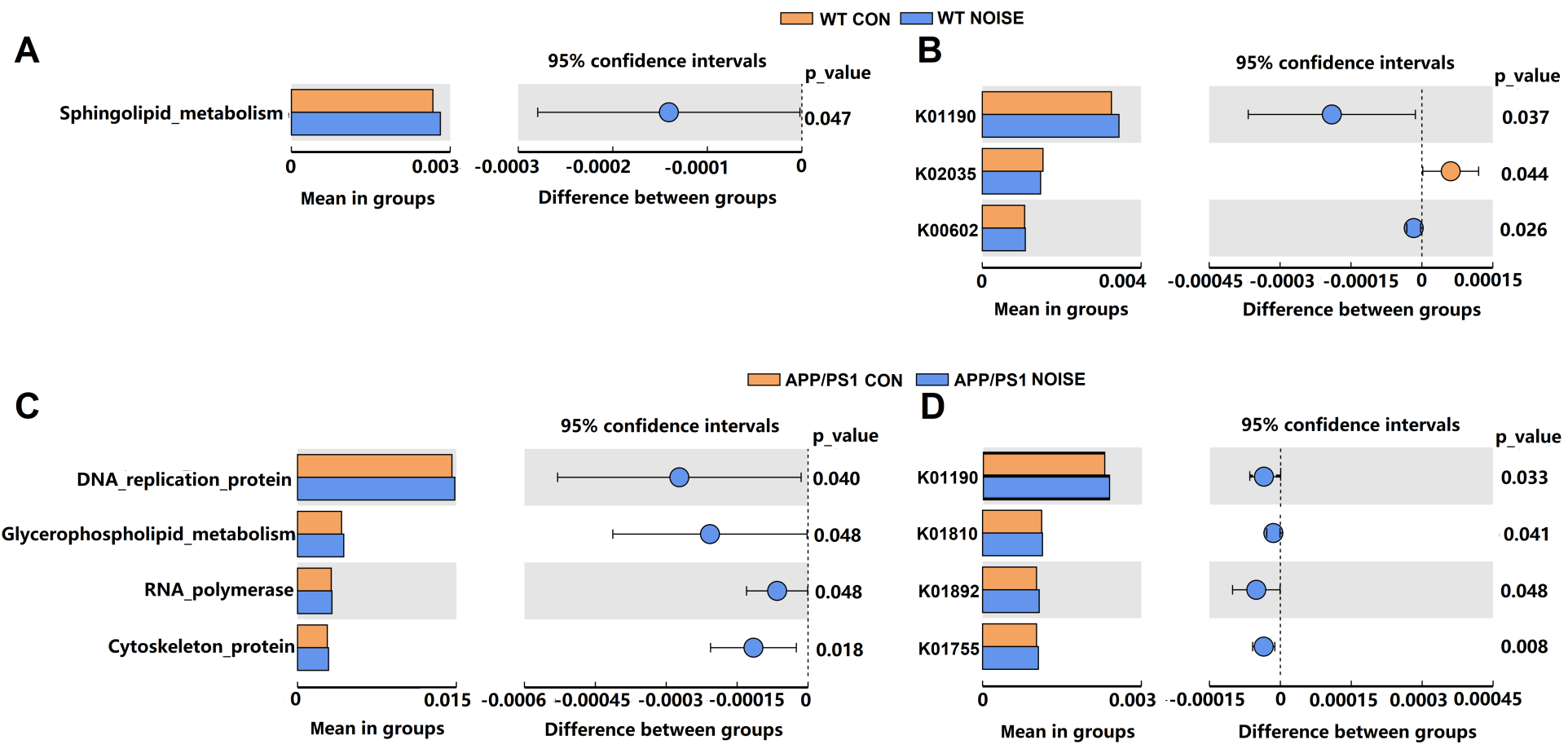

\section{Figure 3}

\section{Figure 3}

Tax4Fun showing predicted functional capabilities of microbial communities based on 16S rRNA datasets. (A) KOs with significant difference at level 3 between the WT CON group and WT NOISE group. (B) KOs with significant difference at level $\mathrm{K}$ between the WT CON group and WT NOISE group. (C) KOs with significant difference at level 3 between the APP/PS1 CON group and APP/PS1 NOISE group. (D) KOs with significant difference at level $\mathrm{K}$ between the APP/PS1 CON group and APP/PS1 NOISE group. CON, control groups; NOISE, noise-exposed groups; WT, wild type mice; APP/PS1, APP/PS1 transgenic mice. 
O CTR " NOISE
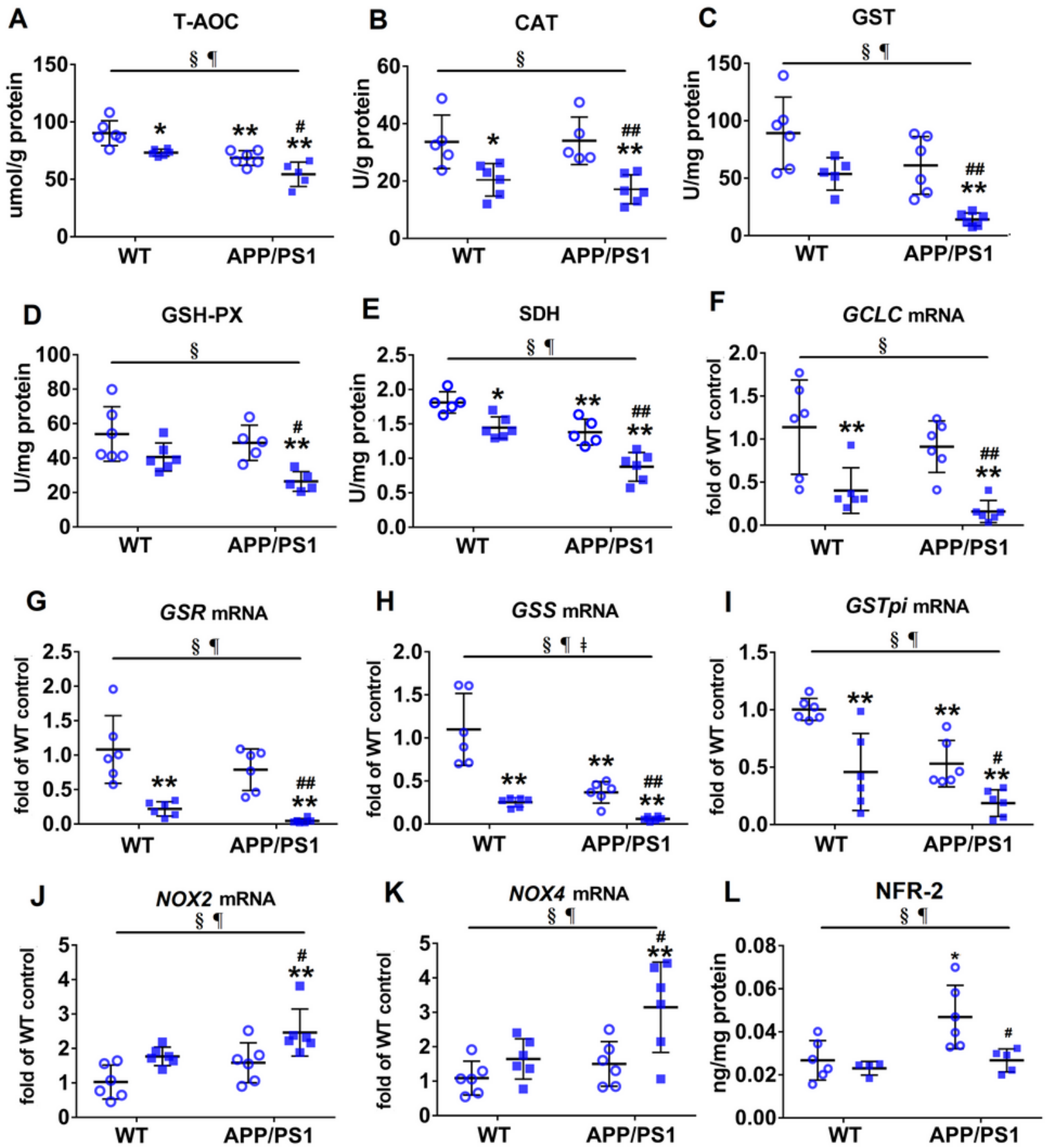

\section{Figure 4}

Figure 4

Effects of chronic noise exposure on the status of oxidative stress in the intestine. (A-E) Antioxidant capacities in cecal contents ( $n=5-6)$. (F-K) mRNA expression levels of key enzymes in GSH/GST pathways and NOX2/4 in colon tissue $(n=6)$. (L) Enzyme-linked immunosorbent assay analysis of Nrf2 content in colon tissue $(n=5-6)$. Data are presented as the mean \pm standard deviation. ${ }^{\star} P<0.05$, $* * P<$ 0.01 vs. WT CON group; \# P<0.05, \#\#P<0.01 vs. APP/PS1 CON group. §: exposure effect, $P<0.05 ; \mathbf{q}$ : 


\section{O CTR - NOISE}
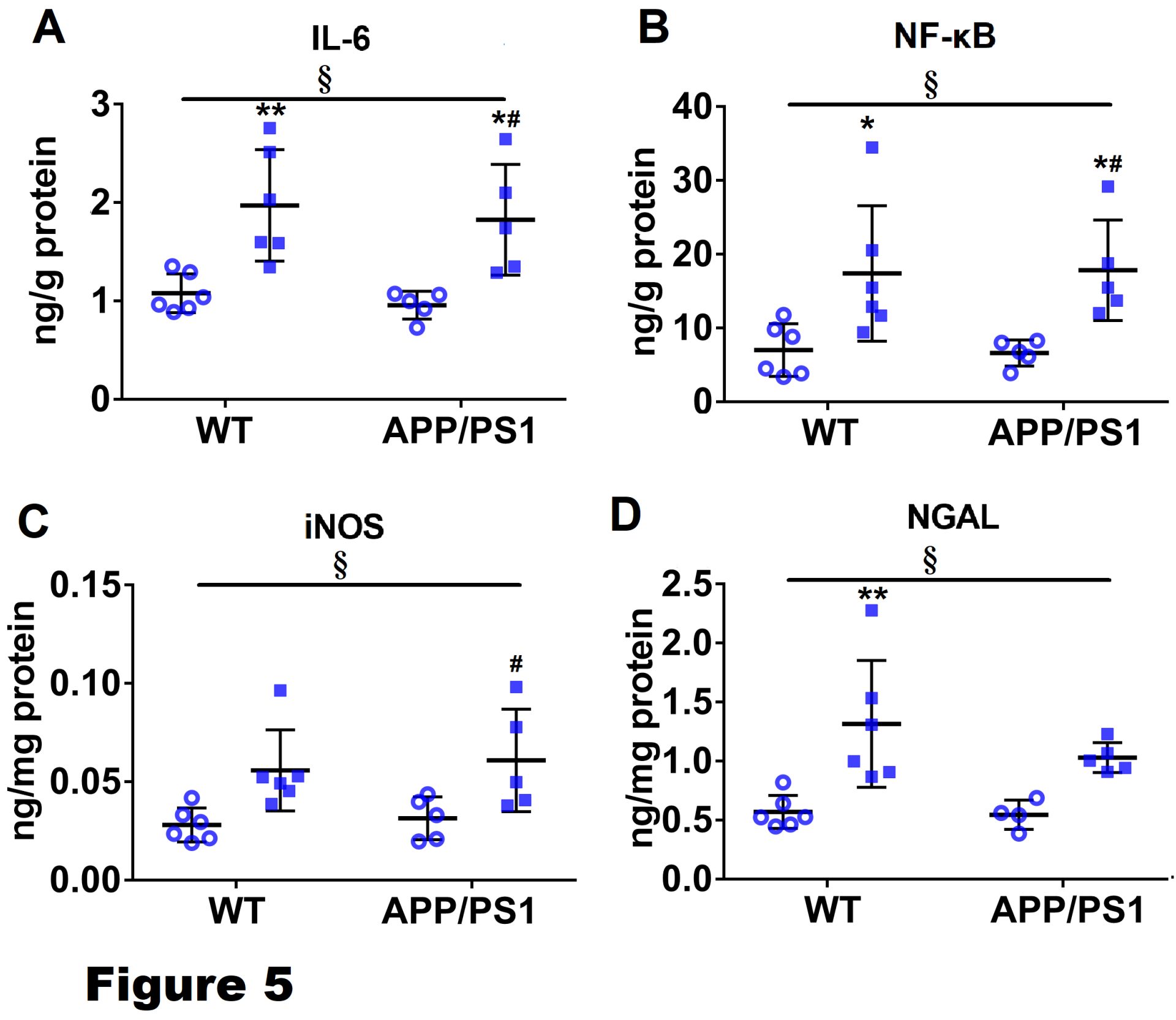

Figure 5

Intestinal abnormalities of host inflammatory responses following noise exposure. (A-D) Enzyme-linked immunosorbent assay analysis of inflammatory mediators (IL-6, NF-KB, iNOS, and NGAL) content in each group $(n=5-6)$. Data are presented as the mean \pm standard deviation. ${ }^{\star} P<0.05,{ }^{*} \mathrm{P}<0.01$ vs. WT CON group; \# P<0.05, \#\#P<0.01 vs. APP/PS1 CON group. §: exposure effect, $P<0.05$; 9 : genotype effect,

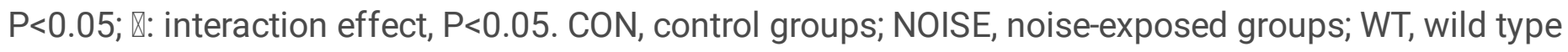
mice; APP/PS1, APP/PS1 transgenic mice. 
O CTR NOISE
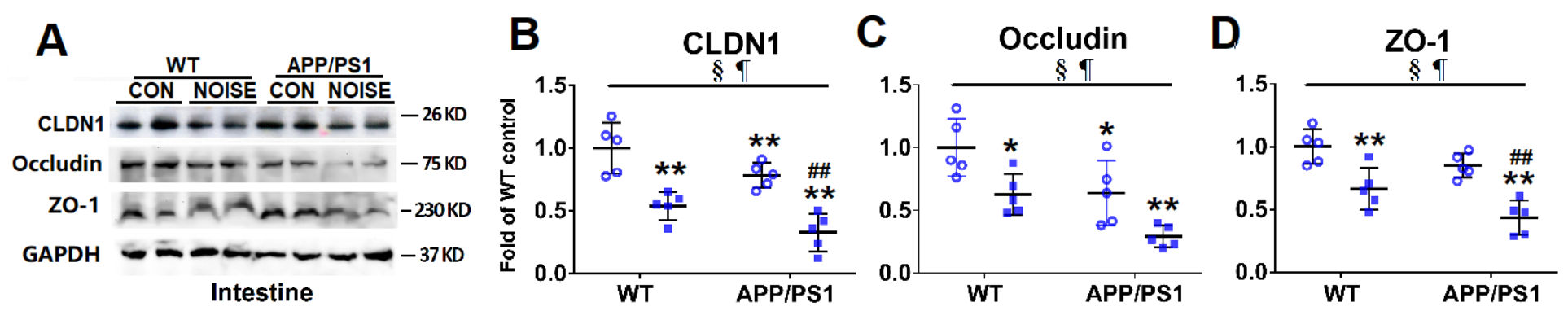

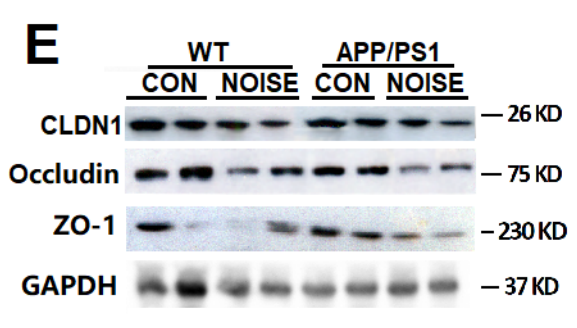

Hippocampus
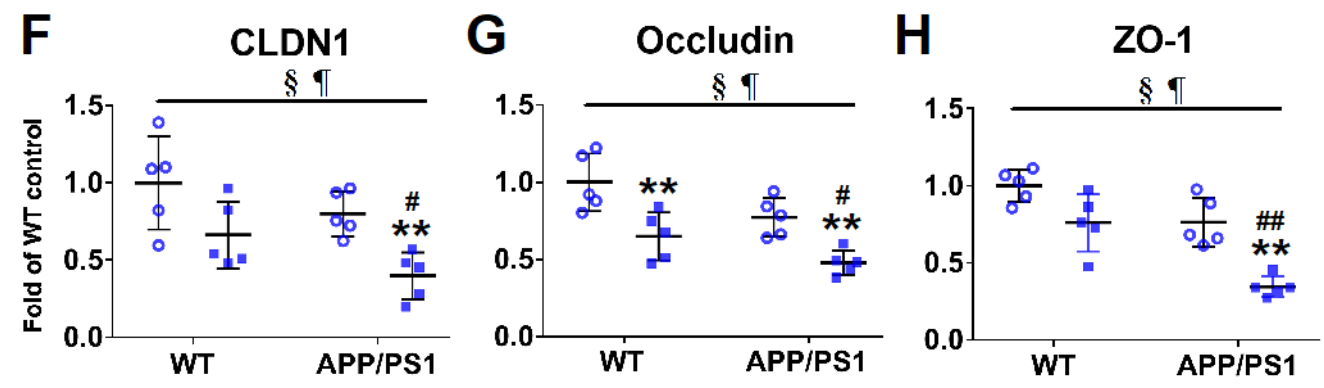

\section{Figure 6}

Figure 6

Chronic noise exposure diminishes tight junction protein expression in the intestine and hippocampus of WT and APP/PS1 mice. (A-D) Protein expression levels of main tight junction components in intestine samples $(n=5)$. $(E-H)$ Protein expression levels of main tight junction proteins in the hippocampus $(n=$ 5). GAPDH was used as a loading control. Data are presented as the mean \pm standard deviation. $* \mathrm{P}<$ 0.05 , ${ }^{\star} \mathrm{P}<0.01$ vs. WT CON group; \# $\mathrm{P}<0.05$, \#\#P < 0.01 vs. APP/PS1 CON group. §: exposure effect, $\mathrm{P}<0.05$; 9 : genotype effect, $\mathrm{P}<0.05$; : interaction effect, $\mathrm{P}<0.05$. CON, control groups; NOISE, noiseexposed groups; WT, wild type mice; APP/PS1, APP/PS1 transgenic mice. 
○ CTR - NOISE
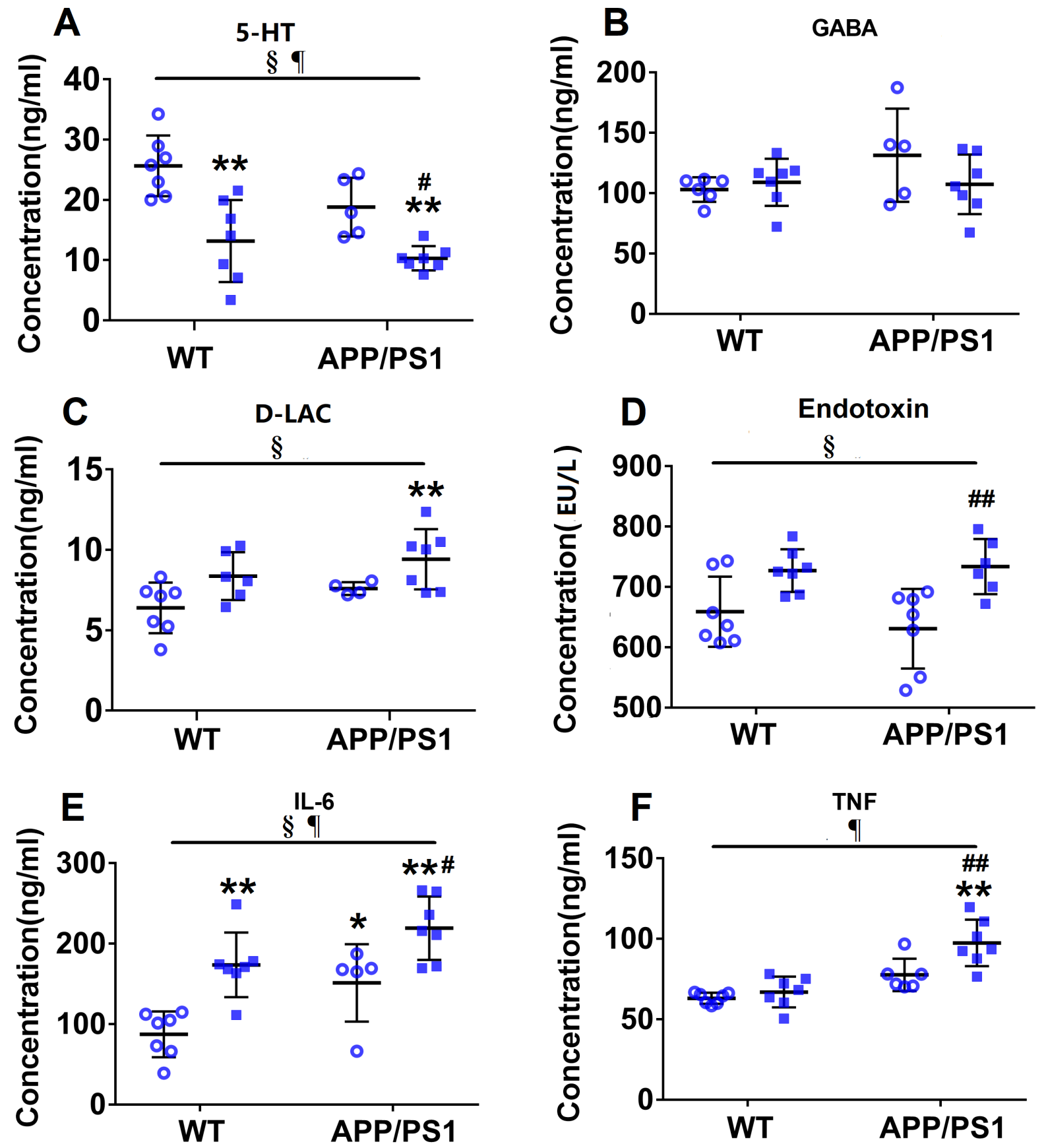

\section{Figure 7}

Figure 7

Circulatory abnormalities in neurotransmitters and inflammatory mediators following noise exposure. (A-

F) Enzyme-linked immunosorbent assay analysis of the concentrations of neurotransmitters (5-HT and GABA) and inflammatory mediators (D-LAC, endotoxin, IL-6, and TNF) in each group ( $n=5-7)$. Data are presented as the mean \pm standard deviation. ${ }^{*} \mathrm{P}<0.05$, ${ }^{\star *} \mathrm{P}<0.01$ vs. WT CON group; $\# \mathrm{P}<0.05$, \#\#P<

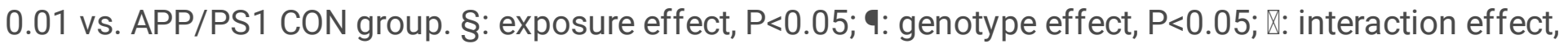


$P<0.05$. CON, control groups; NOISE, noise-exposed groups; WT, wild type mice; APP/PS1, APP/PS1 transgenic mice.

\section{O CTR NOISE}
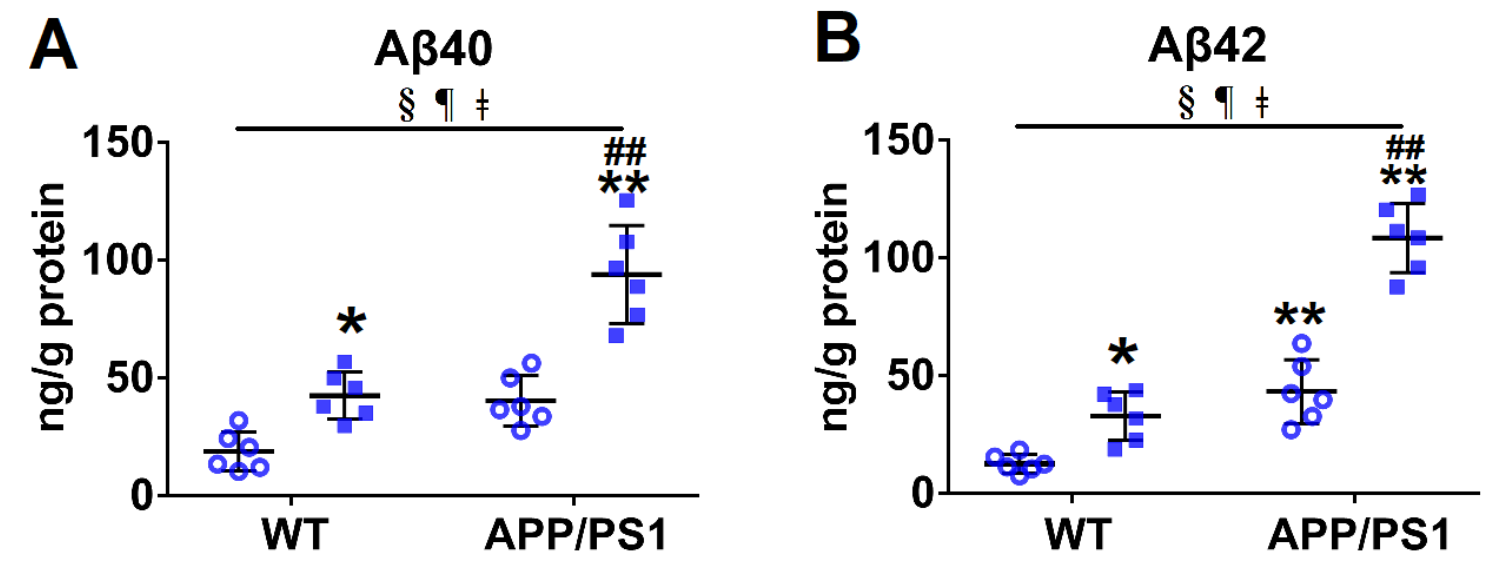

C

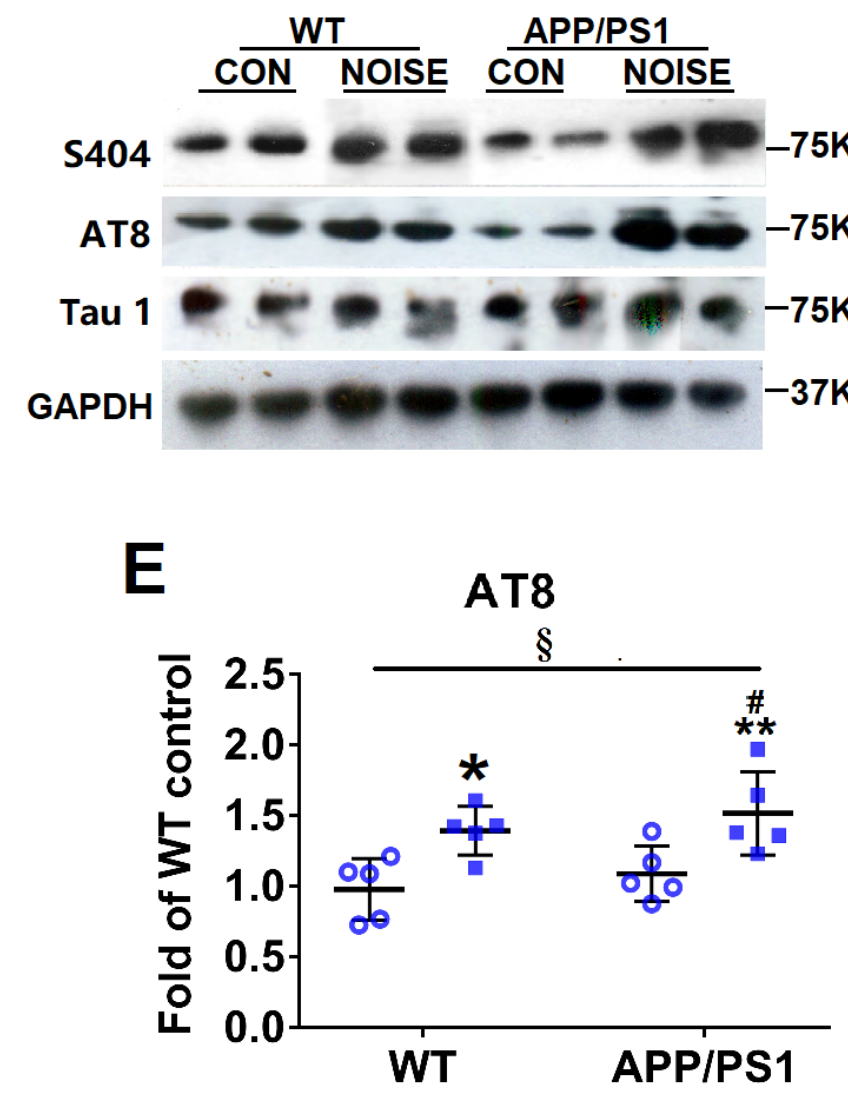

D

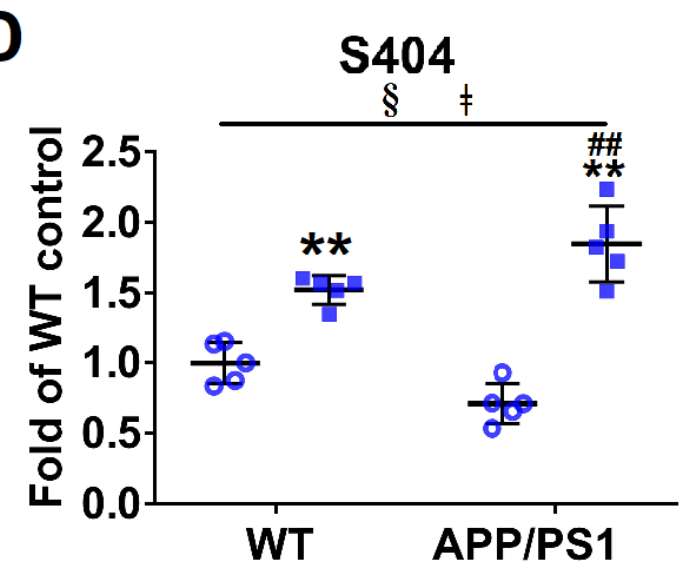

F

Tau1

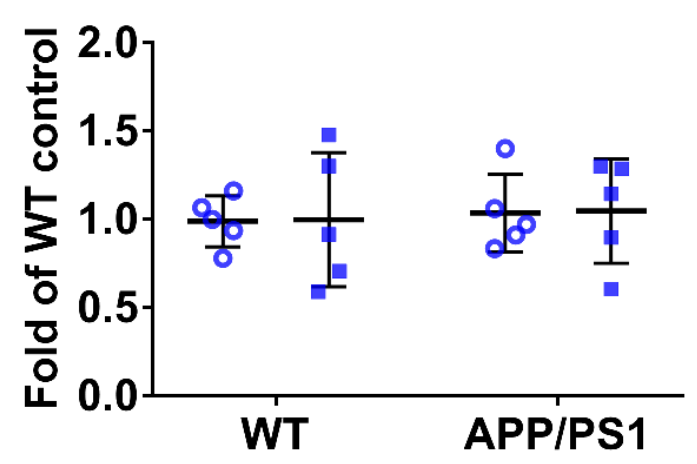

\section{Figure 8}

Figure 8

Chronic noise-induced Alzheimer's disease-like neuropathological alterations in WT and APP/PS1 mice. $(A, B)$ Enzyme-linked immunosorbent assay analysis of $A \beta 40$ and $A \beta 42$ content in each group $(n=6)$. (C-F) Western blot analysis (C) and quantification (D-F) of phosphorylated tau at AD-related sites in the 
hippocampus in each group $(n=5)$. GAPDH was used as a loading control. Data are presented as the percent change relative to control samples $(n=6)$. All data are presented as mean \pm standard deviation. ${ }^{*} \mathrm{P}<0.05,{ }^{*} \mathrm{P}<0.01$ vs. WT CON group; \# $\mathrm{P}<0.05$, \#\#P<0.01 vs. APP/PS1 CON group. §: exposure effect, $\mathrm{P}<0.05$; 9 : genotype effect, $\mathrm{P}<0.05$; $\mathbb{\otimes}$ : interaction effect, $\mathrm{P}<0.05$. CON, control groups; NOISE, noiseexposed groups; WT, wild type mice; APP/PS1, APP/PS1 transgenic mice. 